\title{
Cardiorenal Anemia Syndrome and Survival among Heart Failure Patients in Tanzania: A Prospective Cohort Study
}

\author{
Pedro Pallangyo ${ }^{1 *}$, Francis Fredrick², Smita Bhalia ${ }^{1}$, Paulina Nicholaus ${ }^{1}$, Peter Kisenge ${ }^{1}$, Benjamin Mtinangi ${ }^{2}$,
} Mohamed Janabi ${ }^{1}$ and Stephen Humphrey ${ }^{2}$

\begin{abstract}
Background: Cardiorenal anemia syndrome (CRAS) is an evolving global epidemic associated with increased morbimortality and cost of care. The management of patients with CRAS remains a challenging undertaking worldwide and the lack of evidence-based clinical guidelines adds to the challenge. We aimed to explore the prevalence and survival rates of heart failure patients with CRAS in Tanzania.

Methods: We screened 789 patients and consecutively recruited 463 who met the inclusion criteria. Each participant underwent an interview, physical examination, anthropometric measurements, anemia, renal functions and echocardiographic assessment. All participants were followed until death or for up-to 180 days, whichever came first. Bivariate comparison and subsequent Cox proportional-hazards regression model were used to compare the CRAS and non-CRAS groups with respect to the primary end point.

Results: The mean age of participants was $46.4 \pm 18.9$ years, and $56.5 \%$ were women. Overall, $51.9 \%$ of participants had renal insufficiency, $72.8 \%$ were anemic and $44.4 \%$ had CRAS. During a mean follow-up of $103 \pm 75$ days, 57.8\% of participants died. Patients with CRAS displayed a higher mortality rate (73.5\%) compared to those free of CRAS (45. $8 \%),(p<0.001)$. During multivariate analysis in a cox regression model of 21 potential predictors of mortality; renal dysfunction (HR 1.9; 95\% Cl 1.0-3.5; $p=0.03$ ), severe anemia ( $\mathrm{HR} 1.8 ; 95 \% \mathrm{Cl} 1.0-3.1 ; p=0.04$ ), hyponatremia (HR 2.2; $95 \% \mathrm{Cl} 1.3-3.7 ; p=0.004)$ and rehospitalization ( $\mathrm{HR} 4.3 ; 95 \% \mathrm{Cl} 2.2-8.4 ; p<0.001)$ proved to be the strongest factors.

Conclusion: Cardiorenal anemia syndrome is considerably prevalent and is associated with an increase in mortality amongst patients with heart failure. In view of this, timely, aggressive and collaborative measures to improve renal functions and/or correct anemia are crucial in the management of CRAS patients. Furthermore, these findings call for guideline committees to revise and/or develop evidence-based recommendations for management of patients with CRAS.
\end{abstract}

Keywords: CRAS, Heart failure, Renal insufficiency, Anemia, Mortality

\section{Background}

Comorbid heart failure, renal failure and anemia commonly referred to as cardiorenal anemia syndrome (CRAS) is an evolving epidemic encountered by healthcare professionals worldwide. The interactive links between the CRAS triad are complex and multi-factorial with high potential for increased morbidity, mortality, complexity and cost of care. Studies have reported CRAS

\footnotetext{
* Correspondence: pedro.pallangyo@gmail.com

${ }^{1}$ Department of Cardiovascular Medicine, Jakaya Kikwete Cardiac Institute,

P.O Box 65141, Dar es Salaam, Tanzania

Full list of author information is available at the end of the article
}

prevalence ranging from 19 to $62 \%$ and mortality rates of up-to $51 \%$ amongst heart failure patients [1-8].

The management of patients with CRAS remains a challenging undertaking largely due to the complexity and heterogeneity of this syndrome. Furthermore, the present clinical guidelines lack evidence-based recommendations for managing patients with CRAS. Despite the evidence of increasing hospitalizations due to heart failure and renal dysfunction, CRAS has not been thoroughly explored in Tanzania. To investigate on this subject, we performed a prospective cohort study to determine the prevalence and survival rates of heart failure patients with CRAS. 


\section{Methods}

\section{Recruitment process and definition of terms}

From March through October 2014, we screened for and consecutively enrolled patients with clinical diagnosis of heart failure who were admitted to Muhimbili National Hospital and the Jakaya Kikwete Cardiac Institute in Dar es Salaam, Tanzania. Demographic, clinical, laboratory, and echocardiographic data were gathered during the hospital admission of enrollment. We employed the following prospective selection criteria. Patients were included if they had symptoms of heart failure as per the Framingham criteria and later patients underwent a 2-dimensional echocardiography for diagnosis confirmation. We used an ejection fraction (EF) of below $45 \%$ to denote a systolic dysfunction. Renal dysfunction and anemia were diagnosed using the serum creatinine $(\mathrm{sCr})$ and hemoglobin $(\mathrm{Hb})$ measurements respectively. We estimated the renal functions with the Modification of Diet in Renal Disease (MDRD) equation, where estimated glomerular filtration rate (eGFR) $(\mathrm{mL} / \mathrm{min} /$ $\left.1.73 \mathrm{~m}^{2}\right)=186.3 \times \mathrm{sCr}-1.154 \times$ age $-0.203 \times 0.742$ (if female). No correction for race was necessary as all participants were of African descent. Renal dysfunction was defined by an eGFR value of $<60 \mathrm{~mL} / \mathrm{min} / 1.73 \mathrm{~m}^{2}$. WHO criteria for anemia i.e. $\mathrm{Hb}$ concentration of $<13.0 \mathrm{~g} / \mathrm{dL}$ for males and $<12.0 \mathrm{~g} / \mathrm{dL}$ for females was used to diagnose anemia. Participants were categorized as having CRAS if they had both renal dysfunction and anemia.

\section{Follow-up and study outcomes}

Follow-up continued through April 2015, data was censored at the time of death or after the completion of the 180-day follow-up period, whichever occurred first. Follow-up was conducted through weekly phone calls and a participant was deemed lost to follow-up when all of the 3 phone numbers given during enrollment were not reachable in 3 different occasion 7-days apart, with at least 3 attempts on each occasion. The primary outcome measure was the all-cause mortality within 180 days after enrollment.

\section{Statistical analysis}

STATA v11.0 software was utilized in all statistical analyses. We compared categorical variables using the Pearson Chi square tests or Fisher's exact tests; Student's T-test was used in comparison of continuous variables. Bivariate comparison and subsequent Cox proportional-hazards regression model were used to compare the CRAS and non-CRAS groups with respect to the primary end point. Statistically significant variables maintained in the regression final model underwent stepwise and backward selection procedures. The multivariate models were fitted with baseline covariates associated with mortality by bivariate analysis at the $<0.05$ significance level. Wald Chi-Square tests was used to assess for the interaction terms, with $p<0.1$ considered significant. We did not include patients with missing data or those lost to follow-up in the regression analyses. Differences in survival between the two groups were compared using the log-rank test. Hazard ratios with 95\% confidence intervals and $p$-values are reported. All tests were 2-sided, and statistical significance was defined by $p<0.05$.

\section{Results \\ Study population}

Figure 1 shows the study profile (enrollment, follow-up and survival). During the study period, 463 of the 789 screened patients met the inclusion criteria and were recruited into this study. The baseline demographic characteristics were similar amongst study participants regardless of their CRAS status; however, their baseline clinical and laboratory characteristics displayed a different pattern, Table 1 . The mean age of participants was

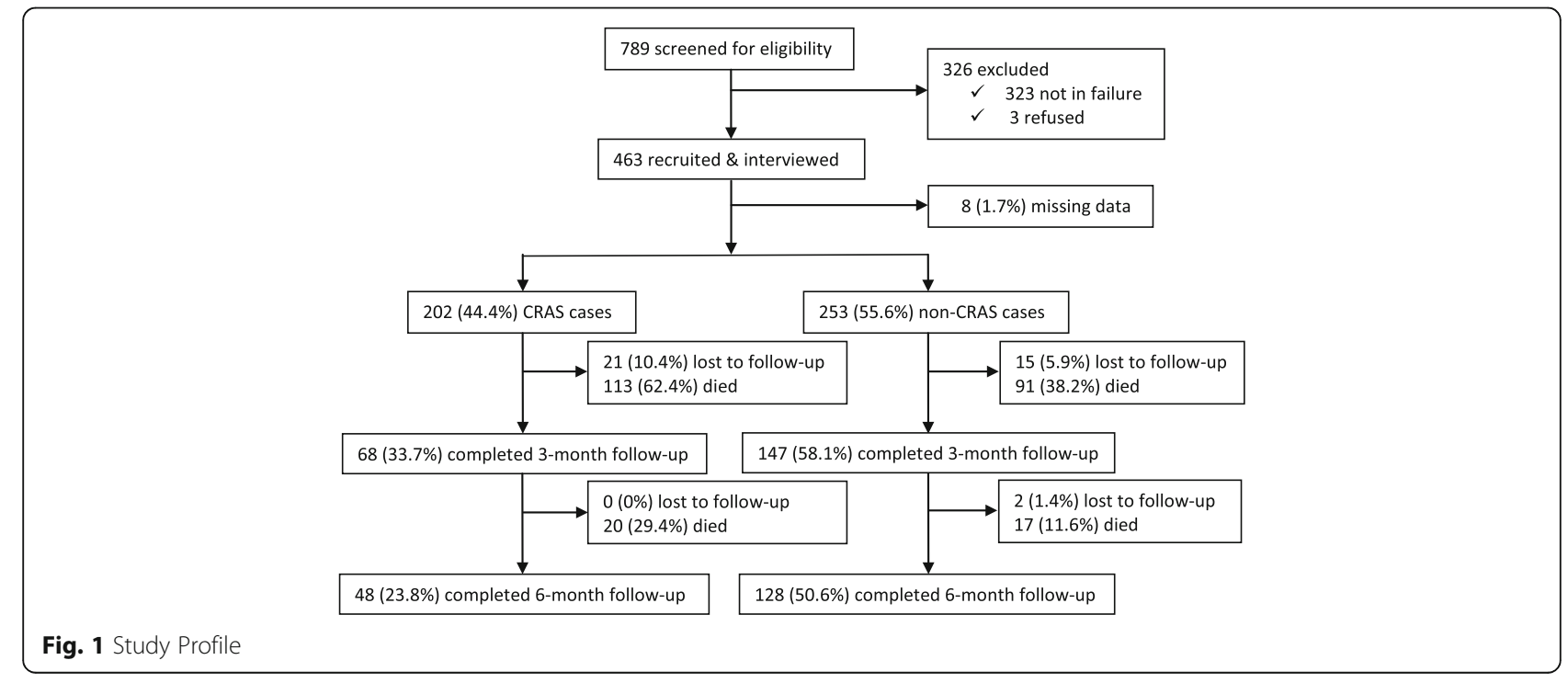


Table 1 Baseline Demographic \& Clinical Characteristics of the Study Population

\begin{tabular}{|c|c|c|c|c|}
\hline Characteristic & All Patients $(n=455)$ & CRAS $(n=202)$ & Non-CRAS $(n=253)$ & $p$-Value \\
\hline \multicolumn{5}{|l|}{ Demographics } \\
\hline - Age, mean (SD), years & $46.4(18.9)$ & $48.1(17.8)$ & $45.0(19.7)$ & \\
\hline - Age groups & & & & 0.09 \\
\hline$<30$ & $112(24.6)$ & $42(20.8)$ & $70(27.7)$ & 0.09 \\
\hline $30-50$ & $140(30.8)$ & $65(32.2)$ & 75 (29.6) & 0.56 \\
\hline$>50$ & $203(44.6)$ & $95(47.0)$ & $108(42.7)$ & 0.35 \\
\hline - Female sex, no (\%) & $257(56.5)$ & $124(61.4)$ & $133(52.6)$ & 0.06 \\
\hline - Primary education (\%) & $319(70.1)$ & $136(67.3)$ & $183(72.3)$ & 0.25 \\
\hline - Self-employment (\%) & $226(49.7)$ & $99(49.0)$ & $127(50.2)$ & 0.80 \\
\hline - Urban residence (\%) & $303(66.6)$ & $132(65.4)$ & $171(67.6)$ & 0.61 \\
\hline - Ever smoker (\%) & $49(10.8)$ & $22(10.9)$ & $27(10.7)$ & 0.95 \\
\hline - Health insurance (\%) & $97(21.3)$ & $41(20.3)$ & $56(22.1)$ & 0.64 \\
\hline \multicolumn{5}{|l|}{ Vital sign, mean (SD) } \\
\hline - Respiratory Rate, breaths/min & $24.1(6.5)$ & $23.9(6.5)$ & $24.3(6.5)$ & 0.48 \\
\hline - Heart rate, beats/min & $98.5(22.2)$ & $94.5(21.0)$ & $101.7(22.7)$ & $<0.001$ \\
\hline - Systolic BP, mmHg & $132.4(35.9)$ & $144.7(40.2)$ & $122.5(28.5)$ & $<0.001$ \\
\hline - Diastolic BP, mmHg & $82.3(24.7)$ & $87.2(26.6)$ & $78.4(22.3)$ & $<0.001$ \\
\hline - Oxygen saturation, \% & $96.3(7.6)$ & $96.5(7.6)$ & $96.2(7.6)$ & 0.64 \\
\hline - NYHA III\&IV (\%) & $420(92.3)$ & $188(93.1)$ & $232(91.7)$ & 0.58 \\
\hline - BMI, kg/m² & $25.1(5.2)$ & $25.6(4.7)$ & $24.8(5.6)$ & 0.13 \\
\hline \multicolumn{5}{|l|}{ Serum concentration, mean (SD) } \\
\hline - Hemoglobin, g/dL & $10.3(3.1)$ & $8.3(2.3)$ & $9.9(2.1)^{a}$ & $<0.001$ \\
\hline - Mean Cell Volume, fL & $82.9(9.0)$ & $81.5(8.4)$ & $84.0(9.4)$ & $<0.01$ \\
\hline - Mean Cell Hemoglobin, pg/cell & $26.6(3.6)$ & $25.9(3.1)$ & $27.1(4.0)$ & $<0.001$ \\
\hline - Creatinine, $\mu \mathrm{mol} / \mathrm{L}$ & $446.3(679.1)$ & $870.1(838.2)$ & $278.2(224.0)^{b}$ & $<0.001$ \\
\hline - Total cholesterol, mg/dl & $4.2(2.2)$ & $5.0(2.4)$ & $3.6(1.8)$ & $<0.001$ \\
\hline - LDL cholesterol, mg/dl & $3.2(2.0)$ & $3.9(2.1)$ & $2.5(1.7)$ & $<0.001$ \\
\hline - HDL cholesterol, mg/dl & $1.0(0.4)$ & $0.9(0.5)$ & $1.0(0.4)$ & 0.25 \\
\hline - Sodium, mmol/L & $132.0(8.3)$ & $131.3(8.8)$ & $132.6(7.9)$ & 0.9 \\
\hline Hyponatremia (\%) & $232(54.7)$ & $112(59.3)$ & $120(51.1)$ & 0.09 \\
\hline - Potassium, mmol/L & $4.4(1.3)$ & $4.9(1.5)$ & $4.1(1.0)$ & 0.9 \\
\hline Hypokalemia (\%) & $80(18.4)$ & $28(14.5)$ & $52(21.6)$ & 0.06 \\
\hline Hyperkalemia (\%) & $130(30.0)$ & $87(45.1)$ & $43(17.8)$ & $<0.001$ \\
\hline - Calcium, mmol/L & $2.1(0.3)$ & $2.1(0.3)$ & $2.1(0.2)$ & 1 \\
\hline Hypocalcemia (\%) & 169 (48.8) & $94(54.3)$ & 75 (43.4) & 0.04 \\
\hline - Magnesium, mmol/L & $0.9(0.2)$ & $0.9(0.2)$ & $0.8(0.1)$ & 1 \\
\hline Hypomagnesemia (\%) & $41(16.1)$ & $14(11.0)$ & $27(21.1)$ & 0.03 \\
\hline \multicolumn{5}{|l|}{ Comorbid conditions } \\
\hline - Hypertension (\%) & $246(54.1)$ & $143(70.8)$ & $103(40.7)$ & $<0.001$ \\
\hline - Diabetes (\%) & $67(14.7)$ & $42(20.8)$ & $25(9.9)$ & $<0.01$ \\
\hline - HIV (\%) & $31(6.8)$ & $18(8.9)$ & $13(5.1)$ & 0.11 \\
\hline Admission Duration, mean, days (SD) & $13.9(13.0)$ & $14.4(11.0)$ & $13.5(14.5)$ & 0.42 \\
\hline
\end{tabular}

${ }^{\mathrm{a}}$ and ${ }^{\mathrm{b}}$ represents anemic $(n=129)$ and renal insufficiency $(n=34)$ subgroups amongst non-CRAS participants respectively 
$46.4 \pm 18.9$ years, and $257(56.5 \%)$ were women. Ninety two point three percent $(92.3 \%)$ of patients were in New York Heart Association (NYHA) functional class III/IV on admission. A total of 246 (54.1\%) participants had a history of hypertension, 67 (14.7\%) had diabetes and 31 (6.8\%) were HIV infected (Additional file 1).

\section{Prevalence and associated factors for CRAS}

Of the 455 participants with complete data, 236 (51.9\%) had renal insufficiency and 331 (72.8\%) had anemia. We found that 202 (44.4\%) of our heart failure cohort had the CRAS. Participants with CRAS displayed a higher likelihood for both end-stage renal disease (ESRD) $(\mathrm{eGFR}<15)$ and the severe form of anemia $(\mathrm{Hb}<8 \mathrm{~g} /$ $\mathrm{dL})$ compared to their non-CRAS counterparts with renal dysfunction or anemia, (both $p<0.001$ ). Comorbid diabetes or hypertension was associated with higher rates of CRAS compared to those free from either condition, $(p<0.01 \& p<0.001$ respectively).

Overall, hypertensive heart diseases (40.9\%) was the leading cause of heart failure, followed by cardiomyopathies $(26 \%)$ and valvular heart diseases $(23 \%)$. Of the valvular etiology, $82.6 \%$ were attributable to rheumatic heart disease. We observed that 114/236 (48.3\%) of participants with renal insufficiency were in ESRD and 14 (12.3\%) of these were on dialysis. Normocytic normochromic anemias constituted 180/331 (54.4\%) of anemias, while the microcytic hypochromic form was found in $107 / 331$ (32.3\%) of anemic subjects.

\section{Primary outcome}

During a mean follow-up of $103 \pm 75$ days, 241/417 (57.8\%) participants died, with $21.6 \%$ of these fatalities occurring either at home or on the way to a health facility. Patients with CRAS had a significantly higher mortality rate $133 / 181(73.5 \%)$ compared to those free of CRAS 108/236 (45.8\%), $(p<0.001)$, Fig. 2 . Mortality rate was observed to increase with worsening of heart failure i.e. $33.3 \%, 54.2 \%$, and $63.2 \%$ in NYHA class II, III, and IV respectively. Presence of anemia and/or renal insufficiency was observed to independently and synergistically increase the mortality rate, Table 2. During multivariate analysis in a cox regression model of 21 potential associated factors for mortality; renal dysfunction (HR 1.9; 95\% CI 1.0-3.5; $p=0.03$ ), severe anemia (HR 1.8; 95\% CI $1.0-3.1 ; p=0.04$ ), hyponatremia ( $\mathrm{HR} 2.2 ; 95 \% \mathrm{CI}$ $1.3-3.7 ; p=0.004)$ and rehospitalization (HR 4.3; 95\% CI $2.2-8.4 ; p<0.001)$ proved to be the strongest factors.

\section{Subgroup analysis}

The superior rate of death observed in participants with CRAS was consistent across all major subgroup analyses we conducted, (Fig. 3). Overall, patients with CRAS

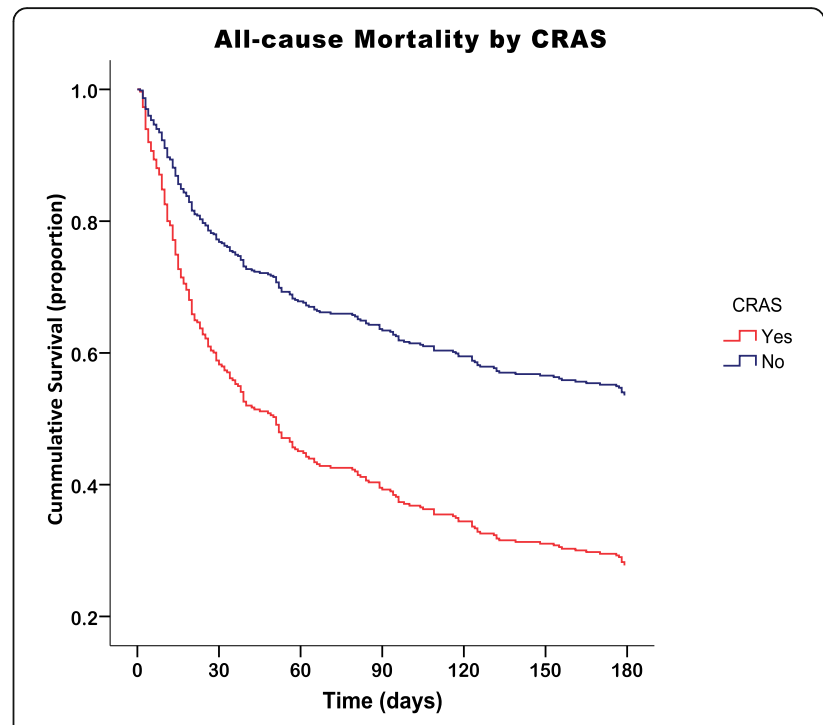

Fig. 2 Cox Proportional Hazard Model Survival Curve by CRAS

displayed a two times mortality rate compared to nonCRAS participants, (HR 2.1, 95\% CI 1.6-2.6, $p<0.001$ ).

\section{Discussion}

The present study shows that CRAS is considerably prevalent and is associated with increased mortality among hospitalized patients with heart failure. These observations are in unison with the previously published data [1-8]. Compared to participants without comorbid renal dysfunction and anemia, patients with CRAS had a 2 -fold mortality hazard. Contrary to previous studies, we observed very high mortality rates within a short period of follow-up. These findings were independent of demographic confounders and established risk factors. Over one fifth of the deceased participants died before reaching a health facility. The authors believe this is so because of the weak emergency systems, poor infrastructure and extreme poverty which hinder a timely access to healthcare services in low and medium countries like Tanzania.

The mean age of our heart failure cohort was 46 years. This is a relatively young age compared to the mean of $>70$ years reported in the western world [9-11]. Such

Table 2 Mortality Rates (\%) by CRAS Triad Components at 30, 90 \& 180 Days

\begin{tabular}{lccc}
\hline & 30-day & 90-day & 180-day \\
\hline HF & 22.7 & 31.3 & 39.0 \\
HF + Anemia & 24.6 & 39.7 & 47.5 \\
HF + CKI & 33.3 & 50.0 & 55.9 \\
HF + CKI + Anemia & 41.4 & 62.4 & 73.5 \\
\hline
\end{tabular}

Key: HF Heart Failure, CKI Chronic Kidney Insufficiency 


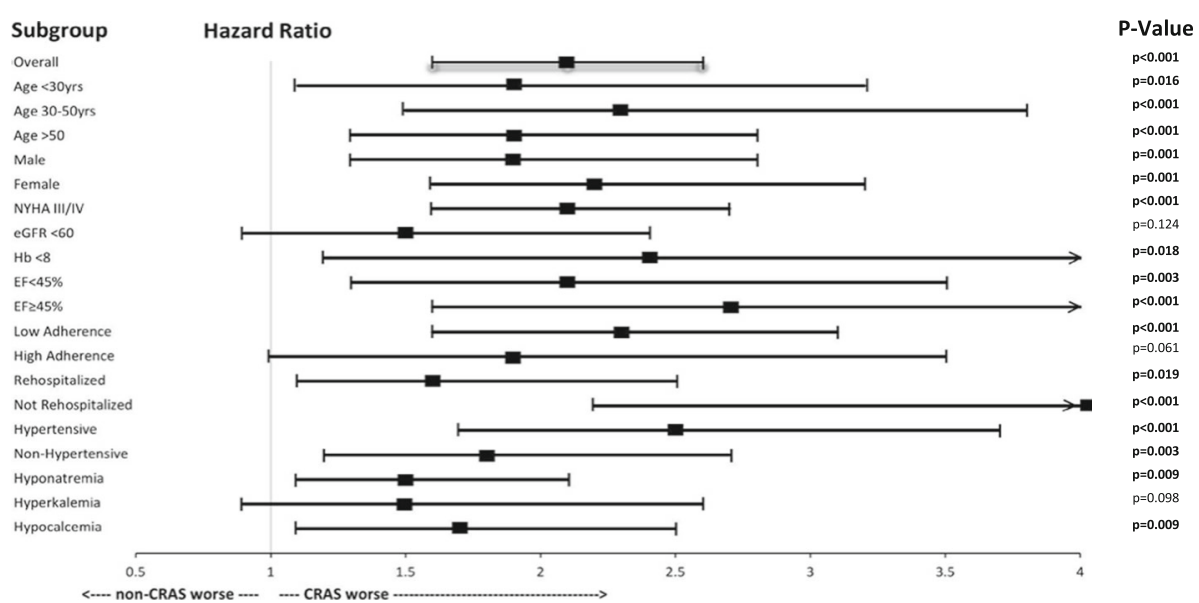

Fig. 3 Hazard Ratios for All-cause Mortality by CRAS. This forest plot shows the hazard ratios (black squares), 95\% Cls (horizontal lines), and p-values for the interaction between the all-cause mortality and any subgroup variable by CRAS-status

young onset of heart failure has been reported in a number of developing nations and remain a major concern. Hypertension continues to be the leading cause of heart failure in resource limited settings. We observed that hypertensive patients had a doubled risk of having CRAS compared to their hypertension-free counterparts, $(\mathrm{RR}=2.1,95 \% \mathrm{CI} 1.6-2.6, p<0.001)$.

Renal insufficiency was present in over a half of the study participants. These findings are in consonance with a number of previous studies [12, 13]. Anemia prevalence and severity was found to increase with worsening of renal functions. These findings are in keeping with our current understanding of anemia's potential in causing renal insufficiency as well as worsening it. Only $12 \%$ of patients in ESRD were on dialysis, this is probably due to the remaining high cost of dialysis despite an increasing number of service providers in this setting.

Almost three-quarters of patients in this present study were anemic. The reported anemia rates among heart failure patients have a wide range among studies and our rate falls within [1]. This variability is partly attributable to the use of inconsistent definitions of anemia (i.e. $\mathrm{Hb}$ cut-off points) and/or different inclusion criteria for age among studies. In this set-up however the anemia prevalence is reportedly high even in the general population almost certainly due to a significant burden of malnutrition and infectious diseases. We therefore hypothesize that a good number of heart failure patients were primarily anemic even before the onset of heart failure.

Our study has several strengths. First, our diagnosis of heart failure though initially utilized a screening tool (Framingham criteria), the diagnostic confirmation relied on a 2-dimensional echocardiography. Enrolled patients were admitted in a tertiary hospital with a national status receiving cases from the whole country and thus our findings are perhaps generalizable to Tanzania and similar resource limited settings. We reported the all-cause mortality which is a relatively unbiased and the most valid endpoint.

This study was observational with obvious limitations. The absence of serial measurements of hemoglobin and creatinine in this study implies that we missed new cases and trends of progression of anemia and renal insufficiency that occurred during the follow-up period. Moreover, with a single creatinine measurement taken, we were not in a position to qualify whether the renal insufficient participants were in acute or chronic phase.

\section{Conclusion}

The CRAS is considerably prevalent and is associated with an increase of mortality amongst patients with heart failure. Our results suggest that in patients with heart failure; timely, aggressive and collaborative measures to improve renal functions and/or correct anemia might improve survival of heart failure patients. Furthermore, these findings call for guideline committees to revise and/or develop evidence-based recommendations for management of patients with CRAS.

\section{Additional file}

Additional file 1: CRAS study Tanzania - dataset. (XLSX $155 \mathrm{~kb}$ )

\section{Abbreviations}

CRAS: Cardiorenal anemia syndrome; EF: Ejection fraction; eGFR: Estimated glomerular filtration rate; ESRD: End stage renal disease; Hb: Hemoglobin; HIV: Human immunodeficiency virus; HR: Hazard ratio; MDRD: Modification of diet in renal disease; NYHA: New York Heart Association; RR: Relative risk; sCr: serum creatinine

\section{Acknowledgement}

We thank the nursing and medical staffs of the Muhimbili National Hospital medical wards and the Jakaya Kikwete Cardiac Institute for their cooperation 
in this study. We extend our gratitude to all the study participants and their relatives for their willingness, tolerance and cooperation offered during the study duration.

\section{Funding}

This study was possible through a research grant from the Commission for Science and Technology (COSTECH) of Tanzania. The investigators and the study sponsor never had confidentiality agreement.

\section{Availability of data and materials}

The final version of data set supporting the findings of this paper is submitted together with this manuscript to the editorial committee. All the raw data is included in this manuscript. There are no ethics restrictions preventing the sharing of the raw data.

\section{Authors' contributions}

PP, FF, BM, and SH conceived the study. PP, SB and PN conducted all the patient recruitment, interviews, and physical examinations. PK and MJ assisted with performing and interpreting echocardiography. PP performed all data entry and analysis. The corresponding author wrote the first draft of the manuscript, and other authors contributed to and approved it. All the authors made the decision to submit the manuscript for publication. All the authors assume responsibility for the accuracy and integrity of the analysis.

\section{Competing interests}

The authors declare that they have no competing interests.

\section{Consent for publication}

Not applicable.

\section{Ethics approval and consent to participate}

The study was approved by the Directorate of Research and Publications of the Muhimbili University of Health and Allied Sciences and the permission to conduct the study was granted by the Muhimbili National Hospital and the Jakaya Kikwete Cardiac Institute. All the participants or caretakers in capacity to consent on behalf, provided written informed consent.

\section{Author details}

'Department of Cardiovascular Medicine, Jakaya Kikwete Cardiac Institute, P.O Box 65141, Dar es Salaam, Tanzania. ${ }^{2}$ Muhimbili University of Health and Allied Sciences, P.O Box 65001, Dar es Salaam, Tanzania.

Received: 10 June 2016 Accepted: 8 February 2017

\section{Published online: 14 February 2017}

\section{References}

1. Scrutinio D, Passantino A, Santoro D, Catanzaro R. The cardio-renal anemia syndrome in systolic heart failure: prevalence, clinical correlates, and long-term survival. Eur J Heart Fail. 2011;13:61-7.

2. Efstratiadis G, Konstantinou D, Chytas I, Vergoulas G. Cardio-renal anemia syndrome. Hippokratia. 2008;12(1):11-6.

3. Schrier R, et al. Cardiorenal syndrome in acute decompensated heart failure. Clin J Am Soc Nephrol. 2009:4:2013-26.

4. Lu KJ, Kearney LG, Hare DL, et al. Cardiorenal Anemia Syndrome as a Prognosticator for Death in Heart Failure. Am J Cardiol. 2013;111(8):1187-91.

5. NP Singh, Kumar A, Sarkar T. cardiorenal syndrome: an overview. Chapter 132. http://www.apiindia.org/medicine_update_2013/chap132.pdf.

6. Geisberg C, Butler J. Addressing the challenges of cardiorenal syndrome. Cleve Clin J Med. 2006;73(5):485-91.

7. Liu PP. Cardiorenal syndrome in heart failure: a cardiologist's perspective. Can J Cardiol. 2008;24:25B-9B.

8. Anand IS. Cardiorenal syndrome: a cardiologist's perspective of pathophysiology. Clin J Am Soc Nephrol. 2013;8(10):1800-7.

9. Gustafsson F, Torp-Pedersen C, Brendorp B, et al. Long-term survival in patients hospitalized with congestive heart failure: relation to preserved and reduced left ventricular systolic function. Eur Heart J. 2003;24:863-70.

10. Cowie MR, Fox KF, Wood DA, et al. Hospitalization of Patients with Heart Failure: A Population Based Study. Eur Heart J. 2002;23:877-85.

11. Lee DS, Austin PC, Rouleau JL, Liu PP, Naimark D, Tu JV. Predicting Mortality Among Patients Hospitalized for Heart Failure: Derivation and Validation of a Clinical Model. JAMA. 2003;290:2581-7.
12. McAlister FA, Ezekowitz J, Tonelli M, Armstrong PW. Renal Insufficiency and Heart Failure - Prognostic and Therapeutic Implications from a Prospective Cohort Study. Circulation. 2004;109:1004-9.

13. Silva R, Nikitin NP, Witte KK, et al. Incidence of Renal Dysfunction over 6 months in Patients with Chronic Heart Failure due to left ventricular systolic dysfunction; contributing factors and relationship to prognosis. Eur Heart J. 2006;27:569-81.

\section{Submit your next manuscript to BioMed Central and we will help you at every step:}

- We accept pre-submission inquiries

- Our selector tool helps you to find the most relevant journal

- We provide round the clock customer support

- Convenient online submission

- Thorough peer review

- Inclusion in PubMed and all major indexing services

- Maximum visibility for your research

Submit your manuscript at www.biomedcentral.com/submit
Biomed Central 